\title{
Observing and Verifying the Quantum Trajectory of a Mechanical Resonator
}

\author{
Massimiliano Rossi, ${ }^{1,2}$ David Mason, ${ }^{1,2}$ Junxin Chen, ${ }^{1,2}$ and Albert Schliesser ${ }^{1,2, *}$ \\ ${ }^{1}$ Niels Bohr Institute, University of Copenhagen, 2100 Copenhagen, Denmark \\ ${ }^{2}$ Center for Hybrid Quantum Networks, Niels Bohr Institute, University of Copenhagen, 2100 Copenhagen, Denmark
}

(Received 26 May 2019; published 14 October 2019)

\begin{abstract}
Continuous weak measurement allows localizing open quantum systems in state space and tracing out their quantum trajectory as they evolve in time. Efficient quantum measurement schemes have previously enabled recording quantum trajectories of microwave photon and qubit states. We apply these concepts to a macroscopic mechanical resonator, and we follow the quantum trajectory of its motional state conditioned on a continuous optical measurement record. Starting with a thermal mixture, we eventually obtain coherent states of $78 \%$ purity - comparable to a displaced thermal state of occupation 0.14 . We introduce a retrodictive measurement protocol to directly verify state purity along the trajectory, and we furthermore observe state collapse and decoherence. This opens the door to measurement-based creation of advanced quantum states, as well as potential tests of gravitational decoherence models.
\end{abstract}

DOI: $10.1103 /$ PhysRevLett.123.163601

Within the Copenhagen interpretation of quantum mechanics, the quantum state of an isolated physical system is represented by its wave function. This mathematical object encodes the probability of possible measurement outcomes and contains the maximum possible knowledge about the system. Under the usually inevitable coupling of the system to an unknown environment, the state evolves into a statistical mixture of quantum states in a process known as decoherence. The mixture is described via a density matrix $\rho$, which again encodes measurement probabilities, while accounting for the ignorance about system-environment interactions. Decoherence entails the disappearance of some of the most salient, and useful, features of quantum mechanics, such as superposition and entanglement. However, if information becomes available on how the system has interacted with the environment, it is possible to restore and retain the purity of the quantum state (i.e., the extent to which the mixture is dominated by a single random but known wave function). Measurements can yield such information; over a finite time interval, however, the obtained information is often incomplete $[1,2]$. The density matrix can anyways be updated, by conditioning on the particular measurement outcome, which purifies the state. Sufficient measurement repetitions can then have the cumulative effect to project the system into a pure quantum state-akin to an ideal von Neumann measurement, which instantaneously collapses

Published by the American Physical Society under the terms of the Creative Commons Attribution 4.0 International license. Further distribution of this work must maintain attribution to the author(s) and the published article's title, journal citation, and DOI. the quantum state into a pure eigenstate of the measurement operator. As the information accumulation through such a weak measurement takes time, obtaining pure conditional states requires measurement rates that approach the system's total decoherence rate. The latter may be notably increased by the presence of the measurement apparatus, which can itself be considered a bath that decoheres the system through its quantum backaction.

In the continuous limit of many weak subsequent measurements carried out over short times, the state conditioned on a measurement record traces out the system's quantum trajectory in time [3]. Observing pure quantum trajectories is a challenging task, so far it has been achieved only in very clean settings such as cavity [4] and circuit $[5,6]$ QED. Here, we extend these ideas to measurements of the motion of a macroscopic mechanical resonator [7-12]. In this setting, pure conditional states are obtained through measurements of high efficiency $\eta_{\text {meas }}=\Gamma_{\text {meas }} /\left(\gamma+\Gamma_{\mathrm{qba}}\right)$, where $\Gamma_{\text {meas }}$ is the measurement rate; and $\gamma$ and $\Gamma_{\mathrm{qba}}$ are decoherence rates induced by a thermal bath and the measurement quantum backaction, respectively $[13,14]$. Prior experiments on motional state estimation have remained confined to the classical regime, due the fast decoherence by the thermal bath [15-18]. In contrast, by probing the system with a measurement for which the efficiency reaches $\eta_{\text {meas }} \approx 67 \%$, we are able to observe individual quantum trajectories of highly pure conditional states. Moreover, building on recent theoretical and experimental work on retrodiction $[10,11,19,20]$ and past quantum states $[21,22]$, we introduce a retrodictionbased trajectory-verification technique, and we use it to confirm the purity of the conditional state by statistical analysis of ensembles of trajectories. This allows us to directly observe the collapse of the conditional state, as 
well as the decoherence that occurs in the absence of information from the measurement.

We explore these ideas in an optomechanical system based on an ultracoherent soft-clamped membrane resonator [23]. The mechanical mode of interest (at the frequency $\Omega_{m} / 2 \pi=1.14 \mathrm{MHz}$ ) corresponds to a localized defect mode created within a phononic crystal. This design simultaneously reduces radiative energy loss and avoids loss-inducing mode curvature, resulting in an extremely low mechanical energy dissipation rate, $\Gamma_{m}$. For the device used here, we find $Q=\Omega_{m} / \Gamma_{m}=1.03 \times 10^{9}$ at temperature $T=11 \mathrm{~K}$. This motion is dispersively coupled to the frequency of a Fabry-Perot cavity mode [linewidth of $\kappa /(2 \pi)=18.5 \mathrm{MHz}]$ at a characteristic vacuum optomechanical coupling rate of $g_{0} /(2 \pi)=129 \mathrm{~Hz}$. Populating the cavity with a large coherent field (with $\bar{n}_{\text {cav }}$ average photons) leads to a linearized field-enhanced coupling at a rate of $g=\sqrt{\bar{n}_{\text {cav }}} g_{0}$. A probe laser that drives this cavity resonantly will acquire phase modulation sidebands proportional to the mechanical displacement, which we detect via a balanced homodyne receiver. The total detection efficiency (including cavity outcoupling) is $\eta_{\text {det }}=74 \%$, ensuring that minimal mechanical information is lost.

In addition to a resonant probe beam, we also utilize an auxiliary beam to provide some precooling of other modes of the membrane, via both sideband and feedback cooling [24]. The effect of this beam on the main mode of interest is simply to change its effective thermal environment. Small, residual detuning of the probe similarly provides some damping. In the following, we account for both of these and refer to the effective energy damping rate and bath occupancy as, respectively, $\Gamma_{m} / 2 \pi \approx 130 \mathrm{~Hz}$ and $\bar{n}_{\text {th }} \approx 2$ such that $\gamma=\Gamma_{m} \bar{n}_{\text {th }} \approx 2 \pi \times 260 \mathrm{~Hz}$.

The quantum measurement backaction of the probe is manifest as radiation pressure force fluctuations, leading to additional mechanical decoherence at a rate of $\Gamma_{\mathrm{qba}}=$ $4 g^{2} / \kappa \approx 2 \pi \times 2.54 \mathrm{kHz}$. Similarly, the mechanical displacement measurement can be characterized by a measurement rate of $\Gamma_{\text {meas }}=4 \eta_{\text {det }} g^{2} / \kappa \approx 2 \pi \times 1.88 \mathrm{kHz}$. Thus, the experimental system studied here can achieve measurements in which quantum backaction dominates thermal motion, and the measurement rate approaches the total decoherence rate (i.e., the measurement efficiency $\eta_{\text {meas }} \rightarrow 1$ ).

The quantum trajectory of the mechanical resonator is derived from quadratures $\mathbf{i}=\left(i_{X}, i_{Y}\right)$ of the homodyne photocurrent, $I(t)$, demodulated at frequency $\Omega_{m}$. This demodulation, which occurs in postprocessing, uses a highorder low-pass filter for which the $\sim 120 \mathrm{kHz}$ bandwidth is significantly larger than the total decoherence rate $\left(\gamma+\Gamma_{\mathrm{qba}} \approx 2 \pi \times 2.80 \mathrm{kHz}\right)$ such that it has a negligible effect on the mechanical signal $[7,25]$. The continuous data stream is subdivided into $3.2 \mathrm{~ms}$ segments: each of which is treated as an individual experimental realization. Examples of the raw photocurrent and one demodulated quadrature are shown in Fig. 1(b).

These photocurrent quadratures form the measurement channels from which we extract the quantum trajectory. This is done according to a stochastic master equation (SME), which simultaneously describes unitary evolution of the system, environmental coupling, and Bayesian updates according to the measurement record [12,26,27]. Note that, although our system is composed of both an optical mode and a mechanical mode, the cavity field can be adiabatically eliminated because $\kappa \gg \Omega_{m}$ [7,28]. For a high- $Q$ resonator, it is also convenient to move to the interaction picture at frequency $\Omega_{m}$ and make a rotating wave approximation. Thus, we describe the system in terms of the slowly varying quadratures $\hat{\mathbf{r}}=(\hat{X}, \hat{Y})$, where the mechanical position is $\hat{q}=\hat{X} \cos \left(\Omega_{m} t\right)+\hat{Y} \sin \left(\Omega_{m} t\right)$. The corresponding SME is [7]

$d \rho=\left(\mathcal{L}_{\text {th }}+\mathcal{L}_{\text {qba }}\right) \rho d t+\sqrt{\Gamma_{\text {meas }}}\left(\mathcal{H}[\hat{X}] \rho d W_{X}+\mathcal{H}[\hat{Y}] \rho d W_{Y}\right)$,

where $\mathcal{L}_{\text {th }}$ and $\mathcal{L}_{\text {qba }}$ describe interactions with the thermal and quantum optical baths, respectively [25]. The final term, written in terms of the measurement superoperator $\mathcal{H}$ and two independent Wiener processes, $\mathbf{W}=\left(W_{X}, W_{Y}\right)$, describes the conditioning of the state upon the measurement record i. We define $\overrightarrow{\mathbf{r}}=\operatorname{tr}(\hat{\mathbf{r}} \rho)$ as the vector of the quadrature expectation values. Assuming Gaussian statistics, the conditional state is fully characterized by these expectation values along with the covariance matrix $V_{i j}=\operatorname{tr}\left(\left\{\hat{r}_{i}-\vec{r}_{i}, \hat{r}_{j}-\vec{r}_{j}\right\} \rho\right) / 2$, where $\{\cdot\}$ is the anticommutator. For our purposes, this covariance can be written in terms of a single number: $V_{i j}=V \delta_{i j}$.

The dynamics of these first and second moments are given by

$$
\begin{gathered}
d \overrightarrow{\mathbf{r}}=-\frac{\Gamma_{m}}{2} \overrightarrow{\mathbf{r}} d t+\sqrt{4 \Gamma_{\text {meas }}} V(t) d \mathbf{W}, \\
\dot{V}(t)=-\Gamma_{m} V(t)+\Gamma_{m}\left(\bar{n}_{\mathrm{th}}+\frac{1}{2}\right)+\Gamma_{\mathrm{qba}}-4 \Gamma_{\text {meas }} V(t)^{2},
\end{gathered}
$$

where the Wiener process is given by the measurement record $d \mathbf{W}=\mathbf{i} d t-\sqrt{4 \Gamma_{\text {meas }}} \overrightarrow{\mathbf{r}} d t$ such that the quantum trajectory as defined in Eq. (2b) represents a filtering of the measurement record $\mathbf{i}$. Note that, although the expectation value evolution is stochastic (driven by the stochastic term $d \mathbf{W}$ ), the conditional variance evolves deterministically, decaying to a steady-state value

$$
V=\frac{\sqrt{1+16 V_{\text {bath }} \Gamma_{\text {meas }} / \Gamma_{m}}-1}{8 \Gamma_{\text {meas }} / \Gamma_{m}},
$$




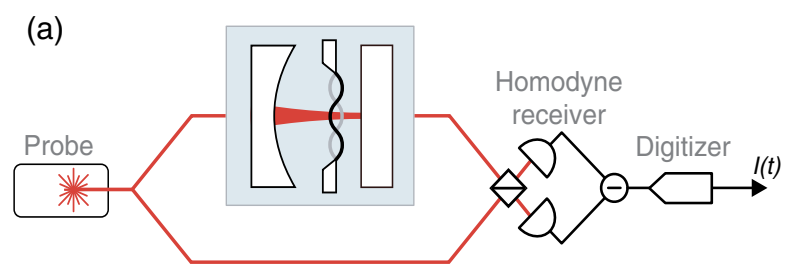

(b)
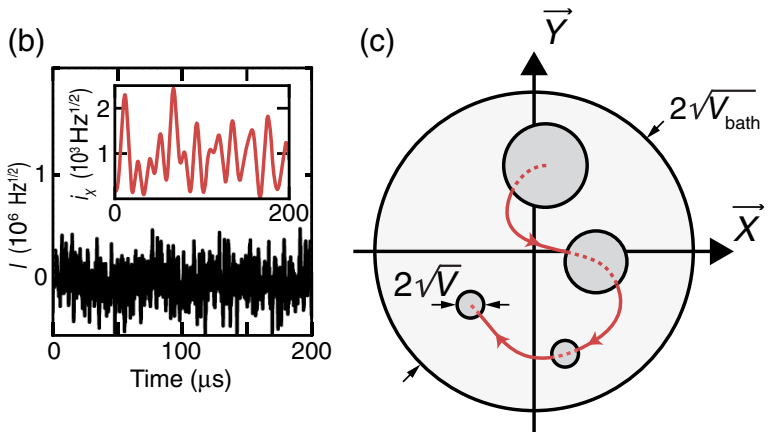

(d)

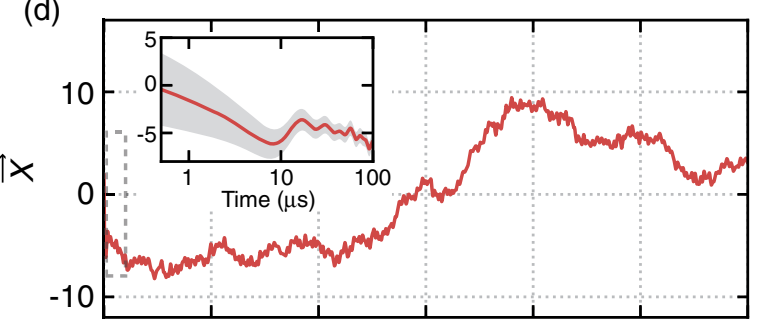

(e)



FIG. 1. Measuring a mechanical quantum trajectory. (a) Experimental setup. The mechanical resonator is coupled to an optical cavity, driven resonantly by a probe laser (red). The motion is imprinted on the phase quadrature of the transmitted light, which is measured with a balanced homodyne detector. The photocurrent $I(t)$ is digitized and analyzed in postprocessing. (b) Example calibrated photocurrent, containing information about all mechanical modes coupled to the cavity. Inset shows one quadrature signal obtained by demodulating the photocurrent at $\Omega_{m}$. (c) Sketch of a quantum trajectory in phase space, in terms of the first moment $\overrightarrow{\mathbf{r}}$ (red line) and conditional variance (dark gray area, standard deviation). The variance is reduced as information is gathered during the measurements. Averaging different realizations together leads to an unconditional, thermal state (light gray area), with variance $V_{\text {bath }}$. (d)-(e) Measured single quantum trajectory $\overrightarrow{\mathbf{r}}(t)$ in terms of slowly varying quadratures, $\vec{X}(t)$ and $\vec{Y}(t)$. Insets illustrate predicted decay of the conditional variance as the conditional state collapses (gray shaded area, standard deviation). where $V_{\text {bath }}=\left(\bar{n}_{\text {th }}+1 / 2+\Gamma_{\mathrm{qba}} / \Gamma_{m}\right)$ is the total bath variance [13]. The reduction of the conditional variance [Fig. 2(a)] to its steady state happens in a characteristic collapse time, which depends inversely on the measurement rate [8]. In the regime of fast, efficient measurement $\Gamma_{\text {meas }} \gg \gamma \gg \Gamma_{m}$, which is relevant to this work, this variance approaches $V \approx 1 /\left(2 \sqrt{\eta_{\text {meas }}}\right)$. Thus, in the limit of highly efficient measurements $\left(\eta_{\text {meas }} \rightarrow 1\right)$, the measurement process is able to project the initial thermal state into a pure coherent state $(V=1 / 2)$. We note that Eqs. (2a) and (2b) are formally equivalent to a Kalman filtering problem [8], with constraints on the measurement and process noises imposed by quantum mechanics. Like the state estimate of a Kalman filter, the quantum state $\rho(t)$ enshrines the most accurate possible prediction of subsequent measurement outcomes.

In Fig. 1(d), we show an example of a quantum trajectory $\overrightarrow{\mathbf{r}}(t)$, as calculated according to Eq. (2a). The region $0<t<100 \mu$ s indicates the collapse time of the measurement; after which, the conditional variance has decayed to its steady-state $V$. For the large probe strength used here (corresponding to $\eta_{\text {meas }}=67 \%$ ), the predicted conditional variance is $V=0.61$, which is only $20 \%$ larger than the zero-point fluctuations. This corresponds to a coherent state with purity $\mathcal{P}=\operatorname{tr}\left(\rho^{2}\right)=1 /(2 V)=0.82$, which is an improvement of almost two orders of magnitude as compared to the initial thermal state purity of $\mathcal{P}=0.02$.

The extracted quantum trajectory depends on the model and the parameters chosen to describe the experiment. Successful modeling of data acquired in earlier experiments testifies to a good understanding of our system, as well as reliable methods to extract its key parameters [24]. We can gain further confidence in the validity of the state estimation by analyzing the experimental innovation function $\mathbf{v} d t=\mathbf{i} d t-\sqrt{4 \Gamma_{\text {meas }}} \overrightarrow{\mathbf{r}} d t$. For correct modeling, this function represents a white noise with a flat power spectral density [16]. This matches the assumption, made above, that the measurement record is driven by a Wiener process. With all system parameters estimated independently, we have calculated the innovation function and checked that, indeed, it has a flat spectrum [25].

Unlike $\vec{r}$, the conditional variance $V$ cannot be immediately obtained from the experimental data: averaging individual trajectories approximates only the unconditional variance, $V_{\text {bath }}$ in this regime [25] (see Fig. 1). To actually verify the prepared conditional state at time $t_{0}$, an experimenter could make a strong projective measurement at that time. Here, we approximate this by a positive-operator valued measure (POVM) measurement based on subsequent data collected for $t>t_{0}[20,21]$. To do so, we backpropagate an effect operator $E$ from future times to the past time $t_{0}$. The role of the effect operator is to refine, in a Bayesian sense, the probabilities for measurement outcomes, as determined by a density matrix $\rho\left(t_{0}\right)$. Together, $\rho$ and $E$ define the past quantum state $[20,21]$ from which the 
expectation value of an operator $\hat{A}$ is calculated as $\operatorname{Tr}(\hat{A} \rho E) / \operatorname{Tr}(\rho E)$. The (unconditional) thermal state could be chosen for $\rho\left(t_{0}\right)$, disregarding the data collected before $t_{0}$. If any prior (to $t_{0}$ ) information about the system is ignored, then $\rho\left(t_{0}\right) \propto \mathbb{1}$ and $E$ contains all information about the quantum state at time $t_{0}$-as determined exclusively from measurements at later times.

For states for which the evolution $\rho(t)$ is restricted to Gaussian states, the effect operator $E$ can be characterized by the expectation value $\overline{\mathbf{r}}=\operatorname{tr}(\mathbf{r} E)$ and the covariance matrix $\left(V_{E}\right)_{i j}=\operatorname{tr}\left(\left\{\hat{r}_{i}-\overleftarrow{r}_{i}, \hat{r}_{j}-\overleftarrow{r}_{j}\right\} E\right) / 2$. For the mechanical measurement performed here, $\mathbf{V}_{\mathbf{E}}$ can be written as $\left(V_{E}\right)_{i j}=V_{E} \delta_{i j}$; and the first and second moments evolve according to $[10,11,20]$

$$
\begin{gathered}
-d \overleftarrow{\mathbf{r}} \equiv \overleftarrow{\mathbf{r}}(t-d t)-\overleftarrow{\mathbf{r}}(t) \\
=\frac{\Gamma_{m}}{2} \overleftarrow{\mathbf{r}} d t+\sqrt{4 \Gamma_{\text {meas }}} V_{E}(t) d \mathbf{W}_{E}, \\
-\dot{V}_{E}(t) \equiv \frac{V_{E}(t-d t)-V_{E}(t)}{d t} \\
=\Gamma_{m} V_{E}(t)+\Gamma_{m}\left(\bar{n}_{\mathrm{th}}+\frac{1}{2}\right)+\Gamma_{\mathrm{qba}}-4 \Gamma_{\text {meas }} V_{E}(t)^{2},
\end{gathered}
$$

where $d \mathbf{W}_{E}=\mathbf{i} d t-\sqrt{4 \Gamma_{\text {meas }}} \leftarrow \mathbf{r} d t$ is a stochastic variable, and the steady-state conditional variance is $V_{E}=$ $V+\Gamma_{m} /\left(4 \Gamma_{\text {meas }}\right) \approx V$. It is this retrodicted trajectory (determined only by the measurement record after $t_{0}$ ) that we will use to verify individual quantum trajectories such as the one shown in Fig. 1.

In Fig. 2(a), we show predicted and retrodicted trajectories, $\overrightarrow{\mathbf{r}}(t)$ and $\overleftarrow{\mathbf{r}}(t)$, in a time interval in which both conditional variances have reached the steady state. These trajectories are compared at an arbitrary common end point, $t_{0}$. Various pairs of $\overrightarrow{\mathbf{r}}\left(t_{0}\right)$ and $\overleftarrow{\mathbf{r}}\left(t_{0}\right)$, from different experimental trials, are shown in Fig. 2. We calculate, over this ensemble of experimental realizations, the covariance matrix of the relative trajectories $\boldsymbol{\sigma}^{2}=\operatorname{Cov}\left[\overrightarrow{\mathbf{r}}\left(t_{0}\right)-\overleftarrow{\mathbf{r}}\left(t_{0}\right)\right]$.

As expected, we always find that $\sigma_{X Y}^{2} \approx 0$ and $\sigma_{X X}^{2} \approx \sigma_{Y Y}^{2}$ (within 2\%), and henceforth report only the average diagonal term, $\sigma^{2}$. This experimental variance provides a direct measurement of the desired quantum conditional variance $V$. As we derive in the Supplemental Material [25], the ensemble variance is given by a simple sum of the variances of the operators characterizing the pre- and retrodicted quantum states, respectively,

$$
\sigma^{2}=V+V_{E} \approx 2 V
$$

which is a result quite compatible with intuition. We experimentally find a variance of $\sigma^{2}=1.29$, which agrees with the predicted $\sigma^{2}=V+V_{E}=1.24$ to within $4 \%$ and
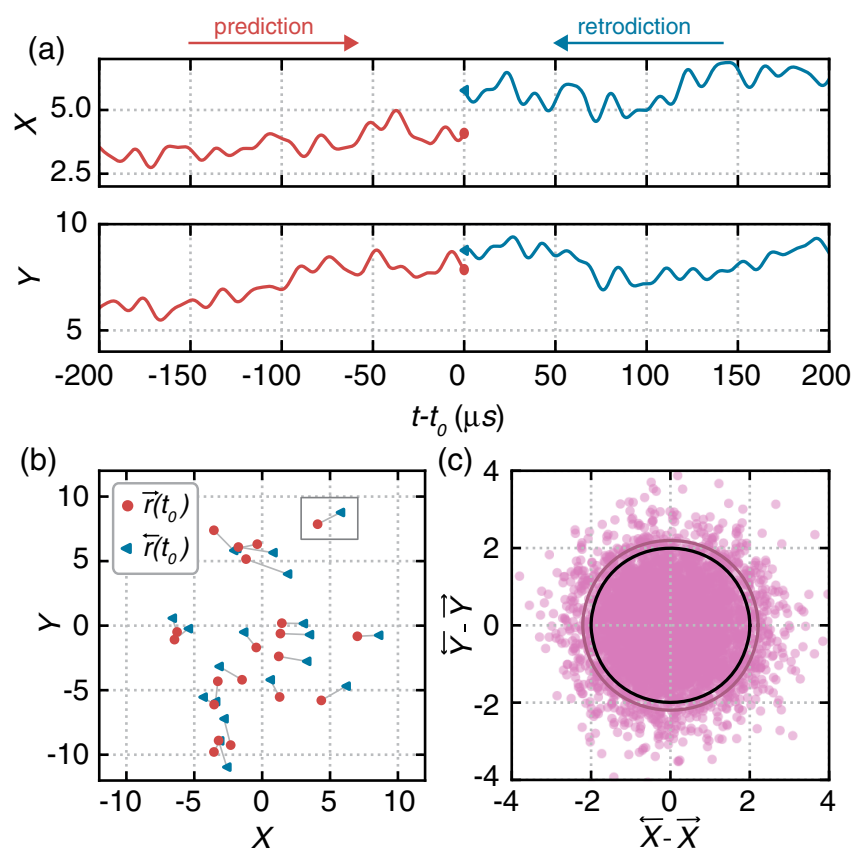

FIG. 2. Verification of the conditional state. (a) A single quantum trajectory, $\overrightarrow{\mathbf{r}}(t)$ (red line), calculated until time $t_{0}$ is compared with a retrodiction, $\overleftarrow{\mathbf{r}}(t)$ (blue line), backpropagated to the same time $t_{0}$. (b) An ensemble of relative state estimations at time $t_{0}$, as measured from the trajectory $\overrightarrow{\mathbf{r}}\left(t_{0}\right)$ (red circles) and verified by the retrodiction $\overleftarrow{\mathbf{r}}\left(t_{0}\right)$ (blue triangles). The predictionretrodiction pairs are connected by a gray line. The gray box indicates the pair shown in Fig. 2(a). (c) Phase space distribution of the relative expectation values: $\overleftarrow{\mathbf{r}}\left(t_{0}\right)-\overrightarrow{\mathbf{r}}\left(t_{0}\right)$ (purple circles). The purple line corresponds to two standard deviations of the data (radius $2 \sqrt{\sigma^{2}}$ ), as compared with the expected $\sqrt{\sigma^{2}}$ for a pure coherent state (black line, radius $2 \sqrt{1}$ ).

corresponds to a purity of $\mathcal{P}=0.78$. (This includes a correction due to a $6 \%$ systematic error introduced by demodulation filter correlations [25]). This corresponds to a displaced thermal state of occupation of $\bar{n}_{\text {cond }}=0.14$. In this sense, this process is sometimes referred to as "cooling by measurement" [15]. Force feedback based on the predicted quantum state can, in principle, entirely undo the displacement to yield a zero-mean low-entropy state $[7,29]$.

We can extend this retrodiction-verification protocol to study the measurement process in more detail, including its dynamics. In particular, we can observe both the measurement-induced collapse of the conditional state and decoherence in the absence of measurement conditioning. To do so, we compare retrodicted quadrature values with the forward-calculated ones at a time $t_{0}$, which now varies within the interval $0<t_{0}<3 \mathrm{~ms}$. The resulting relative trajectories $\left[\overrightarrow{\mathbf{r}}\left(t_{0}\right)-\overline{\mathbf{r}}\left(t_{0}\right)\right]$ and their ensemble variance are shown in Figs. 3(a) and 3(b), respectively. Note that the retrodiction always begins at $3.2 \mathrm{~ms}$ such that its conditional variance is in the steady state throughout the displayed time interval. In contrast, the predictions all 

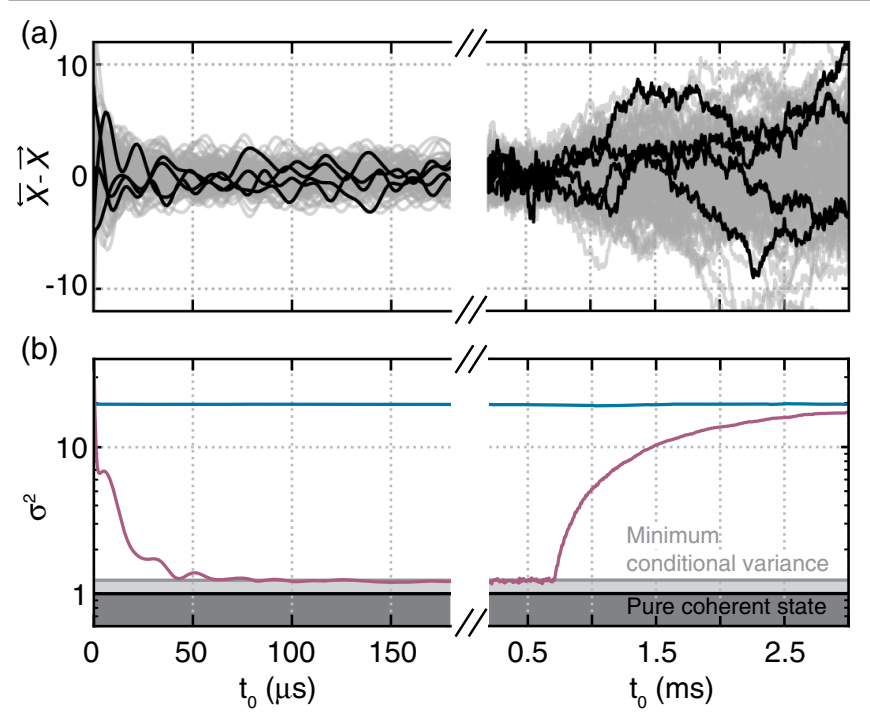

FIG. 3. Measurement-induced collapse and decoherence. (a) $X$ quadratures of relative trajectories $\overleftarrow{\mathbf{r}}\left(t_{0}\right)-\overrightarrow{\mathbf{r}}\left(t_{0}\right)$ (gray lines) shown during different time intervals. A few traces are highlighted in black for illustration purposes. (b) Relative variance $\left(\sigma^{2}\right.$, purple) of the ensemble of relative trajectories from Fig. 3(a). For comparison, the unconditional variance [calculated from $\overleftarrow{\mathbf{r}}(t)$ only] is shown in blue. The theoretical expectation $\left(V+V_{E}\right)$ is shown in gray, and the variance of a pure coherent state is shown in black.

begin at $t=0 \mu \mathrm{s}$. Thus, up to $\approx 50 \mu \mathrm{s}$, the conditional variance $V(t)$ is expected, per Eq. (2b), to decay from an unconditional thermal state. Indeed, this is exactly the behavior revealed by the retrodictive state verification in Fig. 3(b). Next, to visualize the dynamics of the system in the absence of measurement, we set the measurement efficiency to zero in Eq. (2a) from a time of $0.7 \mathrm{~ms}$ onward. That is, we stop conditioning the predicted quantum trajectory based on the measurement record. Thus, the conditional variance rethermalizes, and our nearly pure state decoheres into a statistical mixture.

The ability to effect strong projective displacement measurements via continuous weak measurement opens the door to various measurement-based protocols. Applying the same underlying machinery to modified measurement schemes allows, for example, the production of conditional mechanical squeezed states, as well as conditional entanglement and quantum steering $[10,11,30]$. Moreover, although the analysis presented here was implemented in the postprocessing of data, there is no fundamental obstacle to implementing this protocol as a real-time filter-e.g., using a field-programmable gate array (FPGA). Real-time feedback that removes the (known) mean displacement can then yield unconditional states with the desired quantum correlations. Finally, the application of an optimal quantum-limited state estimation to such ultracoherent mechanical resonators may be of interest to test and constrain models of spontaneous wave function collapse. Indeed, the resonator in this work already suggests experimental bounds for continuous spontaneous localization that are competitive with the state of the art $[25,31,32]$, and the prospects should improve dramatically at millikelvin temperatures.

The authors would like to acknowledge helpful discussions with Klemens Hammerer, Klaus Mølmer, and Marco G. Genoni, as well as sample fabrication by Yeghishe Tsaturyan. This work was supported by funding from the European Unions Horizon 2020 Research and Innovation Programme (European Research Council project Q-CEOM, Grant Agreement No. 638765; and FET proactive project HOT, Grant Agreement No. 732894), a starting grant from the Danish Council for Independent Research, and the Carlsberg Foundation.

M. R. and D. M. contributed equally to this work.

*albert.schliesser@nbi.ku.dk

[1] V. B. Braginsky and F. Y. Khalili, Quantum Measurement (Cambridge University Press, Cambridge, England, 1992).

[2] H. M. Wiseman and G. J. Milburn, Quantum Measurement and Control (Cambridge University Press, Cambridge, England, 2010).

[3] H. J. Carmichael, An Open Systems Approach to Quantum Optics (Springer, New York, 1993).

[4] C. Guerlin, J. Bernu, S. Deléglise, C. Sayrin, S. Gleyzes, S. Kuhr, M. Brune, J.-M. Raimond, and S. Haroche, Progressive field-state collapse and quantum non-demolition photon counting, Nature (London) 448, 889 (2007).

[5] K. W. Murch, S, J. Weber, C. Macklin, and I. Siddiqi, Observing single quantum trajectories of a superconducting quantum bit, Nature (London) 502, 211 (2013).

[6] S. J. Weber, A. Chantasri, J. Dressel, A. N. Jordan, K. W. Murch, and I. Siddiqi, Mapping the optimal route between two quantum states, Nature (London) 511, 570 (2014).

[7] A. C. Doherty, A. Szorkovszky, G. I. Harris, and W. P. Bowen, The quantum trajectory approach to quantum feedback control of an oscillator revisited, Phil. Trans. R. Soc. A 370, 5338 (2012).

[8] A. C. Doherty, S. M. Tan, A. S. Parkins, and D. F. Walls, State determination in continuous measurement, Phys. Rev. A 60, 2380 (1999).

[9] W. P. Bowen and G. J. Milburn, Quantum Optomechanics (CRC Press, Boca Raton, FL, 2016).

[10] J. Lammers, State preparation and verification in continuously measured quantum systems, Ph.D. thesis, Leibniz Universität Hannover, 2018.

[11] J. Lammers and K. Hammerer (to be published).

[12] K. Jacobs and D. A. Steck, A straightforward introduction to continuous quantum measurement, Contemp. Phys. 47, 279 (2006).

[13] M. Aspelmeyer, T. J. Kippenberg, and F. Marquardt, Cavity optomechanics, Rev. Mod. Phys. 86, 1391 (2014).

[14] A. A. Clerk, M. H. Devoret, S. M. Girvin, F. Marquardt, and R. J. Schoelkopf, Introduction to quantum noise, 
measurement, and amplification, Rev. Mod. Phys. 82, 1155 (2010).

[15] M. R. Vanner, J. Hofer, G. D. Cole, and M. Aspelmeyer, Cooling-by-measurement and mechanical state tomography via pulsed optomechanics, Nat. Commun. 4, 2295 (2013).

[16] W. Wieczorek, S. G. Hofer, J. Hoelscher-Obermaier, R. Riedinger, K. Hammerer, and M. Aspelmeyer, Optimal State Estimation for Cavity Optomechanical Systems, Phys. Rev. Lett. 114, 223601 (2015).

[17] G. I. Harris, D. L. McAuslan, E. Sheridan, Y. Sachkou, C. Baker, and W. P. Bowen, Laser cooling and control of excitations in superfluid helium, Nat. Phys. 12, 788 (2016).

[18] A. Setter, M. Toroš, J. F. Ralph, and H. Ulbricht, Real-time Kalman filter: Cooling of an optically levitated nanoparticle, Phys. Rev. A 97, 033822 (2018).

[19] D. Tan, S. J. Weber, I. Siddiqi, K. Mølmer, and K. W. Murch, Prediction and Retrodiction for a Continuously Monitored Superconducting Qubit, Phys. Rev. Lett. 114, 090403 (2015).

[20] J. Zhang and K. Mølmer, Prediction and retrodiction with continuously monitored gaussian states, Phys. Rev. A 96, 062131 (2017).

[21] S. Gammelmark, B. Julsgaard, and K. Mølmer, Past Quantum States of a Monitored System, Phys. Rev. Lett. 111, 160401 (2013).

[22] T. Rybarczyk et al., Forward-backward analysis of the photon-number evolution in a cavity, Phys. Rev. A 91, 062116 (2015).

[23] Y. Tsaturyan, A. Barg, E. S. Polzik, and A. Schliesser, Ultracoherent nanomechanical resonators via soft clamping and dissipation dilution, Nat. Nanotechnol. 12, 776 (2017).
[24] M. Rossi, D. Mason, J. Chen, Y. Tsaturyan, and A. Schliesser, Measurement-based quantum control of mechanical motion, Nature (London) 563, 53 (2018).

[25] See Supplemental Material at http://link.aps.org/ supplemental/10.1103/PhysRevLett.123.163601 for the connection between experimental and theoretical expectation values, discussion of systematic errors and estimates about the detectable spontaneous collapse rate in our system.

[26] V. P. Belavkin, Quantum Filtering of Markov Signal with White Quantum Noise, in Quantum Communications and Measurement, edited by V. P. Belavkin, O. Hirota, and R. L. Hudson (Springer, Boston, 1995), pp. 381-391.

[27] L. Diósi, Continuous quantum measurement and Itô formalism, Phys. Lett. A 129, 419 (1988).

[28] S. G. Hofer and K. Hammerer, Entanglement-enhanced time-continuous quantum control in optomechanics, Phys. Rev. A 91, 033822 (2015).

[29] A. C. Doherty and K. Jacobs, Feedback control of quantum systems using continuous state estimation, Phys. Rev. A 60, 2700 (1999).

[30] S. Danilishin, H. Miao, H. Müller-Ebhardt, and Y. Chen, Optomechanical entanglement: How to prepare, verify and "steer" a macroscopic mechanical quantum state? in Proceedings of the First International Workshop on ECS and Its Application to QIS, Tamagawa University, Tokyo, 2012 (Tamagawa University Quantum ICT Research Institute, 2014).

[31] S. Nimmrichter, K. Hornberger, and K. Hammerer, Optomechanical Sensing of Spontaneous Wave-Function Collapse, Phys. Rev. Lett. 113, 020405 (2014).

[32] A. Vinante, M. Bahrami, A. Bassi, O. Usenko, G. Wijts, and T. H. Oosterkamp, Upper Bounds on Spontaneous WaveFunction Collapse Models Using Millikelvin-Cooled Nanocantilevers, Phys. Rev. Lett. 116, 090402 (2016). 